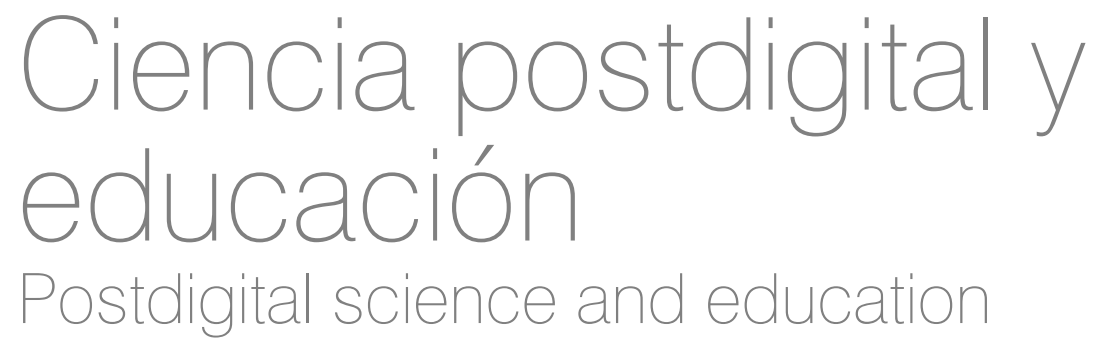

PETAR JANDRIĆ | petar.jandric@tvz.hr
ZAGREB UNIVERSITY OF APPLIED SCIENCES | CROACIA

JEREMY KNOX | jeremy.knox@ed.ac.uk

UNIVERSITY OF EDINBURGH | REINO UNIDO

TINA BESLEY | tbesley@bnu.edu.cn

BEIJING NORMAL UNIVERISTY | CHINA

THOMAS RYBERG | ryberg@hum.aau.dk

AALBORG UNIVERSITY | DINAMARCA

JUHA SUORANTA | juha.suoranta@gmail.com

UNIVERSITY OF TAMPERE | FINLANDIA

SARAH HAYES | Sarah.Hayes@wlv.ac.uk

UNIVERSITY OF WOLVERHAMPTON | REINO UNIDO

Texto original: Petar Jandrić, Jeremy Knox, Tina Besley, Thomas Ryberg, Juha Suoranta y Sarah Hayes (2018). Postdigital science and education, Educational Philosophy and Theory, 50 (10), 893-899. DOI: 10.1080/00131857.2 018.1454000

(C) 2018 Philosophy of Education Society of Australasia. La traducción del artículo ha sido realizada con el permiso de los propietarios del copyright y conserva la misma naturaleza.

NOTA LEGAL DE TAYLOR y FRANCIS :

Artículo con $\odot$ copyright, con permiso de publicación de forma gratuita en la revista Communiars bajo las siguientes condiciones: El permiso se concede únicamente para el uso electrónico no exclusivo de los derechos en idioma español; se debe solicitar permiso para cualquier otro uso. Los derechos electrónicos no permiten que nuestros artículos se vendan como un capítulo individual. Este permiso no cubre ningún trabajo de terceros con derechos de autor que pueda aparecer en el material solicitado. Este permiso no cubre los casos en los que el material de Taylor y Francis consista en más del $20 \%$ de su nuevo trabajo. Debe incluirse el reconocimiento completo con el título del artículo, el autor, el título completo de la revista y los derechos de autor. Philosophy of Education Society of Australasia reimpreso con permiso de Taylor y Francis Ltd, http://www.tandfonline.com en nombre de Philosophy of Education Society of Australasia. No se pueden hacer alteraciones a nuestro trabajo sin consentimiento por escrito. Esta licencia no cubre el contenido de Taylor y Francis que está siendo reeditado en un programa de publicación personalizado o en una base de datos. 
LEGAL NOTE OF TAYLOR y FRANCIS :

Article with $@$ C copyright, with permission to publish free of charge in Communiars journal under the following conditions: Permission is granted for non-exclusive Spanish language rights electronic usage only, permission must be sought for any further use. Electronic rights does not allow our articles to be sold as an individual chapter. This permission does not cover any third party copyrighted work which may appear in the material requested. This permission does not cover cases in which Taylor y Francis material consists of more than $20 \%$ of your new work. Full acknowledgement must be included showing article title, author, full Journal title, copyright (C. Philosophy of Education Society of Australasia reprinted by permission of Taylor y Francis Ltd, http://www.tandfonline.com on behalf of Philosophy of Education Society of Australasia. No alterations may be made to our work without written consent. This license does not cover Taylor y Francis content being republished in a custom publishing program or database.

Enlace del artículo original: https://doi.org/10.1080/00131857.2018.1454000

Traducción de artículo:

Julia Mañero Contreras | julmancon@gmail.com

UNIVERSIDAD DE SEVILLA | ESPAÑA

Recibido: 1 de diciembre de 2018 | Aceptado: 11 de diciembre de 2018

DOI: https://dx.doi.org/10.12795/Communiars.2019.i02.01

Cómo citar este artículo:

Petar Jandrić, Jeremy Knox, Tina Besley, Thomas Ryberg, Juha Suoranta y Sarah Hayes (2019). Postdigital science and education, Educational Philosophy and Theory. Communiars. Revista de Imagen, Artes y Educacion Crítica y Social,2, 11-21.

\section{Ciencia postdigital y educación}

Hemos dejado de pertenecer a un mundo donde la tecnología digital y los medios de comunicación están separados, son virtuales o 'ajenos' a una vida social y 'natural' humana. Este hecho ha inspirado el surgimiento de un nuevo concepto - 'lo postdigital' - el cuál lentamente pero de manera constante está ganando atracción en un amplio rango de disciplinas incluyendo, pero no limitado, a las artes (Bishop, Gansing, Parikka, y Wilk, 2017; Monoskop, 2018), la música (Cascone, 2000), la arquitectura (Spiller, 2009), las humanidades (Hall, 2013; Tabbi, en prensa), ciencias (sociales) (Taffel, 2016), y en muchas otras inter-, trans, y post-disciplinas entre ellas (Berry y Dieter, 2015). A través de está investigación el término postdigital está paulatinamente asentándose en el discurso académico. El Centro de Investigación en Educación Digital de la Universidad de Edimburgo está considerando seriamente renombrarse hacia lo postdigital (Bayne y Jandrić, 2017, p. 204, ver también Jandrić, 2017, p. 201); la Universidad de Coventry recientemente ha fundado el Centro para las Culturas Postdigitales (Coventry University, 2018); los autores de este editorial son editores de la próxima revista de Ciencia Postdigital y Educación¹.

Publicado en la influyente revista Wired, el artículo de 'Beyond Digital' de Nicholas Negropote es una gran fuente de inspiración para el creciente cuerpo de la investigación postdigital y en el cual se reinvindica lo siguiente: "Afrontadlo, la revolución digital ha terminado" (Negroponte, 1998). Esto no significa que lo digital no sea importante. Sin embargo, continua Negroponte, 'en su forma literal, la tecnología ya empieza a darse por 
sentada, y su connotación se convertirá en el compost comercial y cultural del mañana para nuevas ideas'. Al igual que el aire y el agua potable, el hecho de ser digital sólo se notará por su ausencia, no por su presencia" (Negroponte, 1998). En la misma línea, Florian Cramer indica: "lo post-digital" describe un enfoque a los medios digitales que no continúa buscando innovación técnica o mejora, pero considera la digitalización como 'algo que ya ha ocurrido y que podría ser ampliamente reconfigurado’ (Cramer, 2013, ver también Cramer, 2015). En 2013, un amplio grupo de investigadores en transmediale Berlín ha sometido el término a un extenso proceso de revisión por pares el cual ha resultado en una definición de trabajo común:

Lo post-digital, una vez entendido como una reflexión crítica de 'digital' inmaterialismo estético, ahora describe la caótico y condición paradójica del arte y los medios después de la revolución de las tecnologías digitales. Lo 'post-digital' tampoco reconoce la distinción entre los 'antiguos' y 'nuevos' medios, ni una afirmación ideológica de uno o lo otro. Fusiona lo 'antiguo' y lo 'nuevo', aplicando a menudo la experimentación cultural en red a tecnologías analógicas que re-investiga y reutiliza. Tiende a focalizarse más en lo experimental que en lo conceptual. Busca una agencia DIY (Do It Yourself) apartada de la ideología totalitaria e innovadora y la creación de redes a partir del gran capitalismo de datos. Al mismo tiempo, ya se ha comercializado. (Andersen, Cox y Papadopoulos, 2014)

Uno de los primeros libros que trata explícitamente el concepto de postdigital, es de Robert Pepperell y Michael Punt; La Membrana Postdigital: Imaginación, Tecnología y Deseo (2000) e introduce otra definición útil. Para Pepperell y Punt, ‘el término Postdigital tiene la intención de reconocer el estado actual de la tecnología al tiempo que rechaza el cambio conceptual implícito en la "revolución digital", un cambio aparentemente tan abrupto como la "lógica de encendido/apagado" "cero/uno de las máquinas que ahora impregnan nuestra vida cotidiana' (2000, p. 2). Esta definición se focaliza en la diferencia entre la naturaleza continua de la existencia biológica y la naturaleza discreta (encendido/apagado) de la tecnología digital. De esta manera, está fuertemente conectado con el posthumanismo del ciberfeminisno de Donna Haraway (1985/1991), 'la deconstrucción del sujeto humanista liberal' de Katherine Hayles (Potzsch y Hayles, 2014, p. 95) y sus raíces ciberpunks como las de Neuromancia de William Gibson (Gibson, 1984). Esta visión hacia el reto postdigital es particularmente visible en los campos como la educación online (Knox, 2016) y el aprendizaje en red (Jones, Ryberg, y de Laat, 2015), y en preguntas tales como (físicas) identidad (Besley, 2010; Davidsen y Ryberg, 2017; Hayes, 2017), (humano y organizacional) creatividad (Besley y Peters, 2013) y las relaciones entre la tecnología (digital) y los agentes humanos (Hayes, 2015).

La definición de Pepperell y Punt sobre el término postdigital captura algunos aspectos de nuestros momentos históricos donde la centenaria primacía de la física, que ha alcanzado su punto álgido en lo digital, da paso ahora a la biología. Según Dyson,

Ha llegado a ser parte de la sabiduría colectiva decir que el paso siglo XX era el siglo de la física y el siglo XXI será el siglo de la biología. Dos hechos del próximo siglo que son aceptados por casi todos. La biología es ahora más grande que la física, medidas según el tamaño de las inversiones, el tamaño de la fuerza laboral, o por el rendimiento de los descubrimientos mayores; y la biología seguirá siendo probablemente la parte más importante de la ciencia a lo largo del siglo XXI. La biología es también más importante que la física medida por sus consecuencias económicas, por sus implicaciones éticas y por sus efectos en el bienestar humano. (Dyson, 2007) 
Si bien esto puede sonar plausible, el biotecnólogo Craig Venter muestra que la cuestión no es la lucha entre la física / lo digital y la biología / lo analógico. Según Venter,

En realidad, estamos comenzando en un nuevo punto: hemos estado digitalizando la biología y ahora intentamos pasar de ese código digital a una nueva fase biológica con el diseño y sintetización de la vida. Así que hemos estado siempre intentado responder a grandes preguntas. '¿Qué es la vida?' es algo que creo que muchos biólogos han intentado comprender en varios niveles. Hemos intentado varios enfoques reduciéndolos a componentes mínimos. Lo hemos estado digitalizando durante casi 20 años. Cuando secuenciamos el genoma humano, pasaba del mundo analógico de la biología al mundo digital del ordenador. Ahora estamos tratando de preguntarnos: ¿podemos regenerar la vida, o podemos crear nueva vida, a partir de este universo digital? (Venter, 2008)

El reto de digitalizar la biología es técnico y científico. Con unas preguntas éticas emergentes tales como si deberíamos permitir los derechos humanos del genoma y con cambios emergentes en la estructura de la investigación científica incluyendo las incursiones de los datos masivos o macrodatos y los algoritmos, el reto post-digital es también profundo a nivel económico y político. Por lo tanto, Michael Peters desarrolla la noción del capitalismo bioinformacional como 'la forma emergente de la cuarta o quinta generación del capitalismo basado en las inversiones y las devoluciones de estas nuevas bio-industrias: después de los capitalismos mercantil, industrial y de conocimiento', que se 'basa en un código autoorganizado y auto-replicante que aprovecha tanto los resultados de las revoluciones de la información como de las nuevas revoluciones de la biología y los une en una poderosa alianza que mejora y fortalece o refuerza el uno al otro' (Peters, 2012, p. 2). El capitalismo bioinformacional es simultáneamente físico (digital) y biológico (no-digital) (ver también Pierce, 2013). Produciendo profundos problemas éticos y epistémicos como la inmortalidad digital (ver Savin-Baden, Burden, y Taylor, 2017), por lo tanto, es postdigital.

Incluso desde una perspectiva general e incompleta de nuestra literatura, parece intuitivo que el concepto de postdigital parece capturar adecuadamente la existencia humana contemporánea (ver Taffel, 2016, para una visión en conjunto detallada de varias perspectivas de lo postdigital). En estos días, sin embargo, estamos un poco cansados de varios conceptos posteriores, y con razón. En las sociedades post-industriales caracterizadas por la abundancia de bienes de consumo 'no hemos abandonado en absoluto la era de las chimeneas de la producción industrial'. (McLaren y Jandrić, 2014, p. 807, ver también Jandrić, 2017, p. 161). Inmediatamente después de su publicación, el famoso 'el fin de la historia' de Francis Fukuyama (Fukuyama, 1992) ha sido identificado como una construcción ideológica dirigida por la perpetuación interminable del capitalismo (Cox, 2014). El postmodernismo por todas sus tempranas promesas, ha fracaso en conseguir la promesa de superar al modernismo (Peters, 2011). Una línea similar de crítica puede ser aplicada fácilmente a los postdigital (ver Cox, 2014). ¿Por qué inventar un nuevo término cuando no realiza una clara ruptura de las teorías existentes? En respuesta a la crítica de Cox sin embargo, Cramer afirma que el postdentro del postdigital debería ser comprendido de manera diferente. Según Cramer,

El término 'post-digital' puede ser definido de forma más pragmática y significativa dentro de la cultura popular y los marcos de referencias coloquiales. Esto se aplica al prefijo 'post' y al mismo tiempo a la noción de 'digital'. El prefijo 'post' no debería ser entendido aquí bajo el mismo sentido que postmodernismo y post-historia, sino más bien en el sentido del post-punk (una continuación de la cultura punk en formas que de alguna manera siguen siendo punk, 
pero también más allá del punk); post-comunismo (como la realidad socio-política actual en los países del antiguo bloque del Este); post-feminismo (como una continuación críticamente revisada del feminismo, con fronteras borrosas en relación con el feminismo "tradicional", sin fijar); postcolonialismo...y, en menor medida, post-apocalíptico (un mundo en el que el apocalipsis no ha terminado, sino que ha pasado de un punto de ruptura discreto a una condición continua - en términos heideggerianos, de Ereignis a Being - y con una iconografía popular contemporánea iniciada por las películas de Mad Max en los años ochenta). (Cramer, 2015, p. 14)

En el capítulo citado a menudo '¿Qué es "Post-digital"?' Cramer popularmente describe Postdigital 'como un término que apesta pero que es útil' (Cramer, 2015, p. 13).

Quizás debamos volver a las teorías del 'posthumanismo' discutidas previamente para comprender las dimensiones críticas del prefijo 'post-'. También precavido con los 'posts', Nel Badmington describe 'posthumanismo' como 'una taquigrafía conveniente para una crisis general en algo "nosotros" debemos llamar indefectiblemente "humanismo" (2000, p. 2). Así como el humanismo podría haber sido considerado como una especie de calamidad, también podríamos ver que lo 'digital' está pasando por una situación similar. La visión utópica de gratuito, abierto y de comunidad consensuada que caracterizaba en sus inicios a la web parece bastante distante en el clima contemporáneo de poderosas corporaciones de Internet que evitan impuestos, la injerencia política en los medios sociales, el retoque algorítmico de los flujos de medios "personales" y los efectos ambientales de los datos almacenamiento y procesamiento. En la era de la 'post-verdad' (¡si, otra 'post'!) (ver Peters, Rider, Hyvönen, y Besley, 2018) el brillo de la eficiencia, la productividad y la objetividad que una vez pareció caracterizar a lo digital se ha visto empañada por revelaciones de parcialidad, discriminación y desigualdad.

Los amplios problemas sociales para los cuales Silicon Valley ofreció soluciones escurridizas y sencillas fueron quizás demasiado simplistas para comenzar (ver Morozov, 2013), mientras las 'revoluciones' prometidas por los datos masivos y los algoritmos a menudo tendieron a reproducir sus propios y determinados prejuicios (ver, por ejemplo, O'Neil, 2016). Hay una preocupación concreta y creciente acerca de la influencia de lo digital en términos sociales y materiales, que se posiciona en contra de la tendencia a verlo como 'virtual', etéreo, y sin consecuencias 'reales', tal vez captada eficazmente por la conferencia de este año sobre 'Equidad, Rendición de Cuentas y Transparencia'2, centrada en la comprensión de las dimensiones éticas y morales de los sistemas socio-técnicos. Existe por lo tanto un significado adicional y preciado conectado al 'post' de postdigital: un 'tener en cuenta' de lo digital que busca mirar más allá de las promesas de las eficiencias instrumentales, no aclamando su final, pero sobretodo establecer una comprensión crítica de una influencia muy real de estas tecnologías ya que están penetrando cada vez más en la sociedad.

El término postdigital es difícil de definir: desastroso; impredecible; digital y analógico; tecnológico y no-tecnológico; biológico e informacional. Lo postdigital es tanto una ruptura con nuestras teorías existentes como con su continuación. Sin embardo, tal desorden parece ser inherente a la condición humana contemporánea. Por ejemplo, la actual crisis (académica) de publicar resultados de los desastres en las relaciones entre la compresión pre-digital de la propiedad intelectual y las formas digitales de crear y diseminar contenidos (Peters et al., 2016). El reto bien documentado de la mercantilización de la educación no está causada por las tecnologías digitales, sino por sus aspectos principales (incluyendo, pero no limitándose a 
las evaluaciones automáticas) No puede ser concebido sin tecnologías digitales (Hayes y Bartholomew, 2015; Peters, Besley, y Araya, 2013; Peters y Jandrić, 2018). El reto postdigital plantea una importante pregunta epistémica (Suoranta y Vadén, 2010); éstas son particularmente visibles en el campo de los grandes datos y los estudios algorítmicos, y la perspectiva asociada del aprendizaje en red, que apenas ha comenzado a evaluar los resultados y las consecuencias individuales y sociales del mestizaje de la actividad humana y no humana y la capacidad de distinguir claramente entre los dos (Jandrić, Knox, Sinclair y Macleod, 2017; Jones et al., 2015; Knox, 2015, 2018; Ryberg, Sinclair, Bayne y de Laat, 2016).

Tradicionalmente, el campo del aprendizaje en red ha estado caracterizado por un interés particular en lo 'digital' y 'virtual' aspectos traídos por las tecnologías en red, a menudo focalizadas en 'cursos online' con asientos individuales en sus casas, conectados a través de ordenadores de sobremesa a otros alumnos en 'salas virtuales de conferencia'. Sin embargo, es cierto que las redes de aprendizaje contemporáneas están llegando a ser cada vez más diversas,

La omnipresencia del acceso a internet (en algunas partes del mundo) y el dramático aumento de los poseedores de tecnologías móviles (portátiles, tabletas, smartphones) están cambiando los lugares en donde y cómo el aprendizaje en red está ocurriendo. Desde los entornos virtuales de aprendizaje están siendo principalmente usados por la 'educación a distancia' para llegar a ser un componente estándar para todos los estudiantes de educación superior. De ser las TIC y el aprendizaje una actividad esotérica en los laboratorios a convertirse en una parte omnipresente del campus y de las actividades de la sala de conferencias (ya sea conscientemente o no de parte del maestro). De trabajar en principio desde casa a movilizar a personas y comprometerlas en actividades online mientras están en el tren, en cafeterías y alternar a estudiantes entre trabajo distribuido y reuniones en el campo (Ryberg y Sinclair, 2016, p. 13)

Lo anteriormente mencionado se refleja en un aumento del interés en lo sociomaterial, en las prácticas socio-materiales, y en nociones de espacios basados en lugares para el aprendizaje en red (Carvalho, Goodyear, y de Laat, 2016); y, por ejemplo, en explorar los grupos de trabajo de los estudiantes. Ryberg, Davidsen, and Hodgson (2018) advierten de un enfoque demasiado fuerte en 'las tecnologías digitales' que puede que nos haga omitir que las prácticas tecnológicas contemporáneas de los estudiantes son implicaciones complejas entre tecnologías físicas y digitales, espacios, actividades y tiempo. Traduciendo el problemático término 'digital', los investigadores del aprendizaje en red exploran la vanguardia del desafío postdigital.

La llegada del procesamiento intensivo de datos involucra cada vez más lo digital en la supuesta 'humanidad' de la educación, desafiando el punto de vista común de la tecnología como un elemento 'externo' (Bayne, 2014) y cuestionando las creencias a menudo compartidas sobre el proceso de aprendizaje en sí mismo (Knox, 2018). El reto postdigital también se aplica a estudios de trabajo, donde la aceleración social (ver Sinclair, 2017) y las promesas (o tratos) de desempleo tecnológico generalizado puede perturbar significativamente la antigua noción de que los seres humanos se constituyen a sí mismos a través del trabajo (Means, 2017, 2018; Peters, Jandrić, y Hayes, 2018). De este modo, aporta potencial para perturbar décadas de suposiciones lingüísticas que marginalizan el trabajo académico humano en las políticas tecnológicas educativas (Hayes, 2015; Hayes y Bartholomew, 2015). Esto incluye el mito de que la tecnología por si sola tiene un poder innato de efecto positivo, cambios impulsados por el mercado en relación con la manera en que las personas aprenden. Tenemos ante nosotros 
trayectos para resistir estos elementos deshumanizadores de una educación centrada en el consumidor y re-conceptualizar un currículo entrelazado, y nos desvinculado del cuerpo humano (Hayes, 2017).

De hecho, se relaciona estrechamente con relaciones complejas entre la física y la biología, y la noción de Peter del capitalismo bio-informancional (2012). El desafío postdigital va más allá del determinismo tecnológico, investiga futuros alternativos como la igualdad educativa radical (Suoranta y Vadén, 2012) y el cibercomunismo (Vadén y Suoranta, 2009), y busca nuevas oportunidades para las pedagogías críticas (McLaren y Jandrić, 2014 ). Es como si lo postdigital determinase el panorama sociopolítico; sin el 'permiso' de nadie entró en las aulas en los bolsillos de los alumnos y de los profesores (a través de sus dispositivos móviles), inmersos en procesos pedagógicos, y rompió los límites de la enseñanza y el aprendizaje formal e informal: certezas no reflexivas convertidas en incertidumbres reflexivas (Jandrić y Boras, 2015; Peters y Besley, 2015; Vadén y Suoranta, 2007). Así, concluye Cox, 'las rupturas producidas [por el postdigital] no son ni absolutos ni síncronos, sino que funcionan como procesos asíncronos, que ocurren a diferentes velocidades y en diferentes períodos y son culturalmente diversas en cada contexto afectado' (Cox, 2014).

El reto postdigital está alrededor de todos nosotros. En el discurso público desafortunadamente acabó adoptando un nombre que lleva un amargo bagaje de postconceptos anteriores. En consecuencia, el término postdigital puede provocar algunas críticas minuciosas; pero positivamente puede proveer de continuidad histórica ayudándonos a aprender de teorías anteriores y quizás incluso evitar una extraña trampa conceptual. Mirando más allá de la terminología, sin embargo, el uso contemporáneo del término 'postdigital' describe las relaciones humanas hacia las tecnologías que experimentamos, individual y colectivamente, en el momento de aquí y ahora. Muestra nuestra creciente consciencia de las borrosas y desordenadas relaciones entre las físicas y biológicas, antiguos y nuevos medios, humanismo y posthumanismo, capitalismo del conocimiento y capitalismo bio-informacional. Mientras puede que prefiriésemos ir hacia delante con una nueva denominación, percibimos que la condición postdigital es actualmente uno de los grandes retos de la ciencia, la educación, las artes y otras áreas de interés humano. Con todas las imperfecciones, por tanto, acogemos el concepto postdigital y esperamos poder desarrollarlo en el futuro.

\section{REFERENCIAS BIBLIOGRÁFICAS}

Andersen, C. U., Cox, G., y Papadopoulos, G. (2014). Postdigital Research - Editorial. A PeerReviewed Journal About, 3(1).

Badmington, N. (2000). Posthumanism. Basingstoke: Palgrave.

Bayne, S. (2015). What's the matter with 'technology enhanced learning'? Learning, Media and Technology, 40(1), 5-20.

Bayne, S., y Jandrić, P. (2017). From anthropocentric humanism to critical posthumanism in digital education. Knowledge Cultures, 5(2), 197-216.

Berry, D. M., y Dieter, M. (Eds.). (2015). Postdigital aesthetics: Art, computation and design. New York, NY: Palgrave Macmillan UK. 
Besley, T. (2010). Digitized youth: Constructing identities in the creative knowledge economy. En D. Araya y M. A. Peters (Eds.), Education and the creative economy (pp. 485-510). New York, NY: Peter Lang.

Besley, T., y Peters, M. A. (Eds.). (2013). Re-imaging the creative university in the 21st century. Rotterdam: Sense.

Bishop, R., Gansing, K., Parikka, J., y Wilk, E. (Eds.). (2017). Across y beyond: A transmediale reader on post-digital practices, concepts, and institutions. Berlin: Sternberg Press.

Carvalho, L., Goodyear, P., y de Laat, M. (2016). Place-based spaces for networked learning. New York, NY: Routledge.

Cascone, K. (2000). The aesthetics of failure: 'post-digital' tendencies in contemporary computer music. Computer Music Journal, 24(4), 12-18.

Coventry University. (2018). The centre for postdigital cultures (CPC). Recuperado de http://www.coventry.ac.uk/research/areas-of-research/postdigital-cultures/

Cox, G. (2014). Prehistories of the post-digital: Or, some old problems with post-anything. A Peer-Reviewed Journal About, 3(1).

Cramer, F. (2013, 1 de mayo). Post-digital aesthetics. Lemagazine. Recuperado de http://lemagazine.jeudepaume.org/2013/05/florian-cramer-post-digital-aesthetics/

Cramer, F. (2015). What is 'post-digital'? In D. M. Berry y M. Dieter (Eds.), Postdigital aesthetics: Art, computation and design (pp. 12-26). New York, NY: Palgrave Macmillan.

Davidsen, J., y Ryberg, T. (2017). 'This is the size of one meter': Children's bodily-material collaboration. International Journal of Computer-Supported Collaborative Learning, 12(1), 65-90.

Dyson, F. (2007, 19 de julio). Our biotech future. The New York Review of Books. Recuperado de http://www.nybooks.com/articles/2007/07/19/our-biotech-future/\#fnr-*

Fukuyama, F. (1992). The end of history and the last man. New York, NY: Free Press.

Gibson, W. (1984). Neuromancer. New York, NY: Ace Books.

Hall, G. (2013). Towards a post-digital humanities: Cultural analytics and the computational turn to data-driven scholarship. American Literature, 85(4), 781-809.

Haraway, D. ([1985] 1991). Simians, cyborgs, and women: The reinvention of nature. New York, NY: Routledge.

Hayes, S. (2015). Counting on use of technology to enhance learning. In P. Jandrić y D. Boras (Eds.), Critical learning in digital networks (pp. 15-36). New York, NY: Springer.

Hayes, S. (2017). Introducing the concept of 'a corresponding curriculum' to transform academic identity and practice. In A. Horsted, J. Branch, y C. Nygaard (Eds.), Learning centred curriculum design in higher education (pp. 241-274). Faringdon, UK: Libri. 
Horsted, J. Branch, y C. Nygaard (Eds.), Learning centred curriculum design in higher education (pp. 241-274). Faringdon, UK: Libri.

Hayes, S., y Bartholomew, P. (2015). Where's the humanity? Challenging the policy discourse of technology enhanced learning. In J. Branch, P. Bartholomew, y C. Nygaard (Eds.), Technology enhanced learning in higher education (pp. 113-133). London: Libri.

Jandrić, P. (2017). Learning in the age of digital reason. Rotterdam: Sense.

Jandrić, P., y Boras, D. (Eds.). (2015). Critical learning in digital networks. New York, NY: Springer.

Jandrić, P., Knox, J., Sinclair, C., y Macleod, H. (2017). Learning in the age of algorithmic cultures. E-Learning and Digital Media, 14(3), 101-104.

Jones, C., Ryberg, T., y de Laat, M. (2015). Networked learning. In M. Peters (Ed.), Encyclopedia of educational philosophy and theory. Singapore: Springer.

Knox, J. (2015). Critical education and digital cultures. In M. Peters (Ed.), Encyclopedia of educational philosophy and theory. Singapore: Springer.

Knox, J. (2016). Posthumanism and the MOOC: Contaminating the subject of global education. Abingdon: Routledge.

Knox, J. (2018). Beyond the ' $c$ ' and the ' $x$ ': Learning with algorithms in the MOOC. International Review of Education. Recuperado de https://ink.springer.com/content/pdf/10.1007\%2Fs11159-018-9707-0.pdf

McLaren, P., y Jandrić, P. (2014). Critical revolutionary pedagogy is made by walking: In a world where many worlds coexist. Policy Futures in Education, 12(6), 805-831.

Means, A. (2017). Education for a post-work future: Automation, precarity, and stagnation. Knowledge Cultures, 5(1), 21-40.

Means, A. (2018). Learning to save the future: Rethinking education and work in the era of digital capitalism. New York, NY: Routledge.

Monoskop. (2018). Post-digital aesthetics. Monoskop. Recuperado de https://monoskop.org/Post-digital_aesthetics

Morozov, E. (2013). To save everything click here: Technology, solutionism and the urge to fix problems that don't exist. London: Penguin Books Limited.

Negroponte, N. (1998, 12 de enero). Beyond digital. Wired. Recuperado de http://www.wired.com/wired/archive/6.12/negroponte.html

O'Neil, C. (2016). Weapons of math destruction. Bristol: Alan Lane.

Pepperell, R., y Punt, M. (2000). The postdigital membrane: Imagination, technology and desire. Bristol: Intellect. 
Peters, M. A. (2011). The last book of postmodernism: Apocalyptic thinking, philosophy and education in the 21st century. New York, NY: Peter Lang.

Peters, M. A. (2012). Bio-informational capitalism. Thesis Eleven, 110(1), 98-111.

Peters, M. A., y Besley, T. (Eds.). (2015). Paulo Freire: The global legacy. New York, NY: Peter Lang.

Peters, M. A., y Jandrić, P. (2018). The digital university: A dialogue and manifesto. New York, NY: Peter Lang.

Peters, M. A., Besley, T., y Araya, D. (Eds.). (2013). The new paradigm of development: Education, knowledge economy and digital futures. New York, NY: Peter Lang.

Peters, M.A., Jandrić, P., Irwin, R., Locke, K., Devine, N., Heraud, R., ... Stewart, G. (2016). Towards a philosophy of academic publishing. Educational Philosophy and Theory, 48(14), 1401-1425.

Peters, M. A., Jandrić, P., y Hayes, S. (2018). The curious promise of educationalising technological unemployment: What can places of learning really do about the future of work? Educational Philosophy and Theory.

Peters, M. A., Rider, S., Hyvönen, M., y Besley, T. (Eds.). (2018). Post-truth, fake news: Viral modernity y higher education. Singapore: Springer.

Pierce, C. (2013). Education in the age of biocapitalism: Optimizing educational life for a flat world. New York, NY: Palgrave Macmillan.

Pötzsch, H., y Hayles, K. (2014). Posthumanism, technogenesis, and digital technologies: A conversation with N. Katherine Hayles. The Fibreculture Journal, 23, 95-107.

Ryberg, T., y Sinclair, C. (2016). The relationships between policy, boundaries and research in networked learning. In T. Ryberg, C. Sinclair, S. Bayne, y M. de Laat (Eds.), Research, boundaries, and policy in networked learning (pp. 1-20). Cham: Springer.

Ryberg, T., Sinclair, C., Bayne, S., y de Laat, M. (Eds.). (2016). Research, boundaries, and policy in networked learning. Singapore: Springer.

Ryberg, T., Davidsen, J., y Hodgson, V. (2018). Understanding nomadic collaborative learning groups. British Journal of Educational Technology, 49(2), 235-247.

Savin-Baden, M., Burden, D., y Taylor, H. (2017). The ethics and impact of digital immortality. Knowledge Cultures, 5(2), 178-196.

Sinclair, C. (2017). You've got mail: Tracking and framing academic lives. Knowledge Cultures, $5(2), 49-64$.

Spiller, N. (2009). Plectic architecture: Towards a theory of the post-digital in architecture. Technoetic Arts: A Journal of Speculative Research, 7(2), 95-104.

Suoranta, J., y Vadén, T. (2010). Wikiworld. London: Pluto Press. 
Suoranta, J., y Vadén, T. (2012). Wikilearning as radical equality. In P. P. Trifonas (Ed.), Learning the virtual life: Public pedagogy in a digital world (pp. 98-113). New York, NY: Routledge.

Tabbi, J. (en imprenta). Post-digital humanities. London: Bloomsbury.

Taffel, S. (2016). Perspectives on the postdigital: Beyond rhetorics of progress and novelty. Convergence: The International Journal of Research into New Media Technologies, 22(3), 324-338.

Vadén, T., y Suoranta, J. (2007). From social to socialist media: The critical potential of the wikiworld. In P. McLaren y J. Kincheloe (Eds.), Critical pedagogy: Where are we now? (pp. 143-162). New York, NY: Peter Lang.

Vadén, T., y Suoranta, J. (2009). A definition and criticism of cybercommunism. Capital y Class, 33(1), 159-177.

Venter, C. (2008). On the verge of creating synthetic life. [Conferencia TED] Recuperado de https://www.ted.com/talks/craig_venter_is_on_the_verge_of_creating_synthetic_life

\section{Notas}

\footnotetext{
${ }^{1}$ Postdigital Science and Education tiene contrato con Springer, y su primer número se espera para principios de 2019 : https://www.springer.com/education+\%26+language/journal/42438

${ }^{2}$ Ver https://fatconference.org/
} 